Published in "Physica A: Statistical Mechanics and its Applications

391(3): 647-655, 2012"

which should be cited to refer to this work.

\title{
How altruism works: An evolutionary model of supply networks
}

\author{
Zehui Ge $^{\mathrm{a}, \mathrm{b}}$, Zi-Ke Zhang ${ }^{\mathrm{c}, \mathrm{d}, \mathrm{e}, *}$, Linyuan Lü ${ }^{\mathrm{d}, \mathrm{e}}$, Tao Zhou ${ }^{\mathrm{d}, \mathrm{f}}$, Ning Xi $^{\mathrm{a}, \mathrm{b}}$ \\ a Business School, University of Shanghai for Science and Technology, Shanghai, 200093, PR China \\ ${ }^{\mathrm{b}}$ Research Center for Complex Systems Sciences, University of Shanghai for Science and Technology, Shanghai, 200093, PR China \\ ${ }^{\mathrm{c}}$ Web Sciences Center, University of Electronic Science and Technology of China, Chengdu 610054, PR China \\ ${ }^{\mathrm{d}}$ Institute of Information Economy, Hangzhou Normal University - Hangzhou 310036, PR China \\ e Department of Physics, University of Fribourg, Chemin du Musée 3, 1700 Fribourg, Switzerland \\ ${ }^{\mathrm{f}}$ Department of Modern Physics, University of Science and Technology of China, Hefei 230026, PR China
}

\begin{abstract}
Recently, supply networks have attracted increasing attention from the scientific community. However, it lacks serious consideration of social preference in Supply Chain Management. In this paper, we develop an evolutionary decision-making model to characterize the effects of suppliers' altruism in supply networks, and find that the performances of both suppliers and supply chains are improved by introducing the role of altruism. Furthermore, an interesting and reasonable phenomenon is discovered that the suppliers' and whole network's profits do not change monotonously with suppliers' altruistic preference, $\eta$, but reach the best at $\eta=0.6$ and $\eta=0.4$, respectively. This work may shed some light on the in-depth understanding of the effects of altruism for both research and commercial applications.
\end{abstract}

\section{Introduction}

In the last decade, Supply Chain Management (SCM) has made significant strides in both theoretical and practical fronts [1-6]. However, how to manage relationship with suppliers (or retailers, dealers) still remains a huge challenge, since firms have to face the coexistence problem of cooperation and competition with other firms in an interdependent environment. As pointed out by Simchi-Levi et al. [1], supply chain analysis inspires new research ventures that blend operations research, game theory, and microeconomics. However, although quantitative supply chain analysis provides insight into understanding competition and cooperation among firms, there are limitations to understand the nonlinear dynamics and social impacts on free market structures [7]. SCM is the management of a network of interconnected businesses, which suggests that the complexity and adaptivity should not be ignored in a network background [8-11]. In addition, the mainstream research supposes that agents in supply chains intend to make self-maximizing decisions. These operational decisions are on the basis of agents' optimal prediction of the future. However, these so-called "optimal" behaviors could not be easily predictable, sometimes even unbelievable when a large number of agents interact in a complex supply network. Actually, it will be crucial to examine the evolution of supply networks over an extended time horizon [7], e.g., Barabási et al. proposed an evolutionary model of social network's time evolution [12]. Different from the rational focus on the future benefits, they behave dependently on their past behaviors. Furthermore, there are sufficient evidences indicating that firms care about their partners' profits in the chain as well as their own, in order to keep or improve the competitiveness of the total supply chain [13]. For example, Steiner contends that business decision-making today is a mixture of altruism,

\footnotetext{
* Corresponding author at: Web Sciences Center, University of Electronic Science and Technology of China, Chengdu 610054, PR China. E-mail address: zhangzike@gmail.com (Z.-K. Zhang).
} 


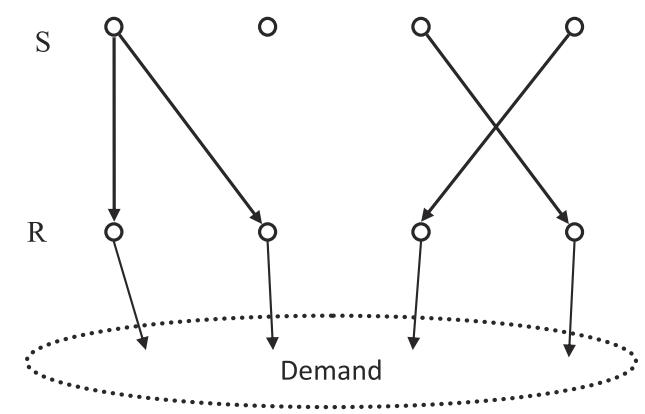

Fig. 1. A simplified supply network, where $S$ and $R$ represent suppliers and retailers, respectively, and arrows represent the direction of physical flows.

self-interest, and good citizenship [14]. At the same time, many behavioral economic researchers have developed various forms of interdependent social preferences to justify experimental observations [15-18]. The main concepts of existing interpretations are based on fairness, or altruism. That is to say, people consider fairness based on relative payoffs, or how monetary payoffs are distributed among them [19-22]. Though Tooby and Cosmides [23] have argued that people may help friends who are unlikely to pay back, DeScioli and Robert empirically demonstrated that the altruism did contribute to the formation of alliance [24]. Moreover, it is also demonstrated that the existence, incentives, and effects of altruism are wildly existing in human behaviors from the perspectives of both social science and biology [25-29].

In this paper, we investigated the effects of altruism on an evolving supply network: how suppliers' altruistic decisionmaking mechanism affects the suppliers' performance from the perspective of evolutionary dynamics [30]. In addition, we proposed a model to evaluate how altruism benefits supply networks, in which an evolutionary pricing mechanism is proposed under the assumption that firms are altruistic when they make decisions. The results show that suppliers have incentives to be altruistic, because the altruistic decision-making mechanism makes them better.

The rest of this paper is organized as following. In Section 2, we describe some settings of our model in Section 2 and the mechanism in Section 3. After this, we present the model results and analysis in Section 4, and conclude in Section 5.

\section{The settings of model}

In this section, we study a supply network in which $n$ retailers order one type of product from $m$ suppliers and sell it to consumers who decide the market demand. In this supply chain from suppliers to the final consumers, the retailers actually play the role of matchmaker with whom the market becomes more efficient [31]. Normally, a retailer can order products from more than one supplier. However, for simplicity, we assume that one retailer is supplied only by one supplier in this paper, ${ }^{1}$ and the quantity that he orders at time $t$ is denoted by $b_{i}(t), i=1,2, \ldots, n$. For a single supplier, he can supply for all the retailers in the market. Fig. 1 shows an example of a simplified supply network.

In the following, we shall first describe the entities in the supply network. Second, the underlying mechanisms of pricing, demand satisfaction and supplier selection are introduced. We finally give decision sequences and present a process for simulations.

\subsection{Suppliers}

In the initial status, each retailer randomly chooses one supplier. At time step $t$, the suppliers respectively propose a wholesale price combination $W(t)=\left[w_{1}(t), w_{2}(t), \ldots, w_{m}(t)\right]$ at time step $t$. Denote the profit of retailer $R_{i}$ at time step $t$ by $\pi_{i}^{r}(t)$, and that of supplier $S_{j}$ by $\pi_{j}^{s}(t)$, respectively. Let $A(t)=\left[a_{i j}(t)\right]$ be an adjacent matrix at time step $t$, in which $a_{i j}(t)=1$ if retailer $R_{i}$ orders from supplier $S_{j}$, otherwise $a_{i j}(t)=0$. Then, $\pi_{j}^{c}(t)=\pi_{j}^{S}(t)+\sum_{i=1}^{N} \pi_{i}^{r}(t) a_{i j}(t)$ indicates a total profit of the supply chain supplied by supplier $S_{j}$, which potentially is a tree with $S_{j}$ as its root. Provided supplier $S_{j}$ 's sold quantity $Q_{j}(t)$, then his profit is

$$
\pi_{j}^{s}(t)=w_{j}(t) Q_{j}(t)-c_{j}^{p} Q_{j}(t), \quad j=1, \ldots, m,
$$

where $c_{j}^{p}$ is unit producing cost of $S_{j}$, randomly determined in the following simulations.

\subsection{Retailers}

Let $p_{i}(t)$ be a retailing price set by retailer $R_{i}(i=1, \ldots, n)$, and $P=\left[p_{1}, \ldots, p_{n}\right]$. As mentioned above, retailer $R_{i}$ faces a newsvendor problem $[32,33]$ with a stochastic demand $D_{i}=D_{i}(P) \xi_{i}$, where $\xi_{i}$ is a random variable $i=1, \ldots, n$. Without loss

\footnotetext{
1 It does not mean the absence of competition among suppliers. Potentially, suppliers compete with each other, since retailers maybe cut their order quantities when they face a high wholesale price.
} 
of generality, assume that $\xi_{i}$ is independent with each other, and $\mathrm{E}\left(\xi_{i}\right)=1, i=1, \ldots, n$, where $\mathrm{E}$ is the expectation function. We suppose $D_{i}(P)$ is a commonly used demand function: multinomial logit choice model [34], which can be written as,

$$
D_{i}(P)=\frac{\alpha_{i} \exp \left(-\beta p_{i}\right)}{1+\sum_{j=1}^{n} \alpha_{j} \exp \left(-\beta p_{j}\right)},
$$

where $\alpha_{i}>0, \beta>0, i=1, \ldots, l$ are constant parameters of consumer choices, and 1 in denominator represents the consumer lost, more details see Ref. [34].

Suppose that retailer $R_{i}$ orders $b_{i}$ units from his supplier, then the units of sold product would be $U_{i}=\min \left\{D_{i}(P) \xi_{i}, b_{i}\right\}$. In each time step, retailers make combined price/inventory decisions and compete for consumers via different pricing strategies. For simplicity, we suppose there is no backorder, and no salvage value for retailers. Thus, the retailer $R_{i}$ 's profit is,

$$
\pi_{i}^{r}(t)=U_{i}(t) \times p_{i}(t)-c_{i}^{h} b_{i}(t)-\sum_{j=1}^{m} b_{i}(t) w_{j}(t) a_{i j}(t),
$$

where $c_{i}^{h}$ is the holding cost, randomly determined in our simulations. Also, his decision-making can be formulated as

$$
\max _{p_{i}(t), b_{i}(t)} \pi_{i}^{r}(t), \quad i=1, \ldots, n .
$$

According to Refs. [33,35], it has been proved that there is a unique solution under a certain condition. ${ }^{2}$

\section{Mechanisms}

\subsection{Wholesale pricing}

At each time step $t$, each supplier $S_{j}$ has to decide whether to change his wholesale price. Denote the incremental price by $\Delta w_{j}(t)=w_{j}(t)-w_{j}(t-1)$. The incremental of $w_{j}$ may bring a change of total profit to the chains supported by $S_{j}$, denoted as $\Delta \pi_{j}^{c}(t)=\pi_{j}^{c}(t)-\pi_{j}^{c}(t-1)$. As discussed in Ref. [15], an altruistic supplier considers his partners' payoff when making decisions. Social preferences systematically affect economic decision making in supply chain transactions.

There is a stream in the literature to model altruistic preference by introducing a proxy into decision makers' utility (e.g., Refs. $[15,19,21]$ in the current manuscript). Here, we incorporate this part of utility into his evolutionary decision process. ${ }^{3}$ Now we describe how suppliers adjust their wholesale prices. Provided with $\Delta w_{j}(t)$, if $\Delta \pi_{j}^{c}(t) \geq 0$, then go ahead and set the wholesale price $w_{j}(t+1)$ such that $\Delta w_{j}(t+1) \times \Delta w_{j}(t) \geq 0$; otherwise, turn around and set $w_{j}(t+1)$ satisfying $\Delta w_{j}(t+1) \times \Delta w_{j}(t) \leq 0$. In all, supplier $S_{j}$ decides $w_{j}(t+1)$ by $^{4}$

$$
w_{j}(t+1)=w_{j}(t)+\eta \frac{\Delta \pi_{j}^{c}(t)}{\pi_{j}^{c}(t)} \Delta w_{j}(t),
$$

where $\eta$ indicates suppliers' altruistic preference, ${ }^{5}$ ranging from 0 to 1 in this paper. In the extremal cases $\eta=0$ and $\eta=1$, the suppliers will exhibit no altruism and full altruism, respectively. As a consequence, the pricing decisions are mainly affected by two aspects: historical decisions and altruistic preference. Given those chains supplied by $S_{j}$, the wholesale price determines how to allocate the total profit. The higher the wholesale price is, the larger share the supplier will get. Thus, $w_{j}(t)$ reflects how to competitively allocate the total pie, while $\Delta w_{j}(t)$ reflects supplier $j$ 's altruistic incentive to make a bigger pie.

\subsection{Supplier selection}

Similar with estimation for non-manufacturer industrials and emerging market in $Z$-score method [36], we generally define retailer $R_{i}$ 's return on investment (brought by the current supplier) as $\gamma_{i}=\sum_{\tau=t_{0}}^{t} \pi_{i}^{r}(\tau) /\left[\sum_{\tau=t_{0}}^{t} b_{i}(\tau) w_{j}(\tau) a_{i j}(\tau)\right]$ for given memory length $\tilde{t}=t-t_{0}$, and the mean return over all retailers by $\bar{\gamma}=\sum_{i=1}^{n} \gamma_{i} / n$. One can see Refs. [37,38] for discussions about memory of past information in various contexts. Let $z_{i}=\left(\gamma_{i}-\bar{\gamma}\right) / \operatorname{std}\left(\gamma_{i}\right)$ represents the distance

\footnotetext{
2 The condition includes three common assumptions in the literature: (1), $\xi_{i}$ is independent of $P$, has an increasing generalized failure rate (see Ref. [2] for details) and a normalized expectation; $(2), D_{i}(P)$ is bounded and differentiable in a closed interval of $P$, i.e., $[P, \bar{P}] ;(3), D_{i}(P)$ is decreasing in $p_{i}$, down to 0 at $p_{i}=\bar{p}_{i}$, but with an increasing price elasticity.

3 In the literature, there are two familiar formulations to characterize decision-maker's utility when altruism is observed (e.g., Refs. [15,21]), $\pi^{s}+\eta \pi^{r}$ and $\eta \pi^{s}+(1-\eta) \pi^{r}$. In essential, they are equivalent with $\pi^{s}+\eta \pi^{c}$.

$4 w_{j}$ will keep constant in the future once $\Delta w_{j}(t)=0$. At this time, we set $\Delta w_{j}(t)=\Delta w_{j}(t-1)$ when $\Delta \pi_{j}^{c}(t)>0$, and set $\Delta w_{j}(t)=-\Delta w_{j}(t-1)$ when $\Delta \pi_{j}^{c}(t)<0$.

5 In this paper, we discuss the average level of altruistic preference in the network, assumed to be identical across agents.
} 


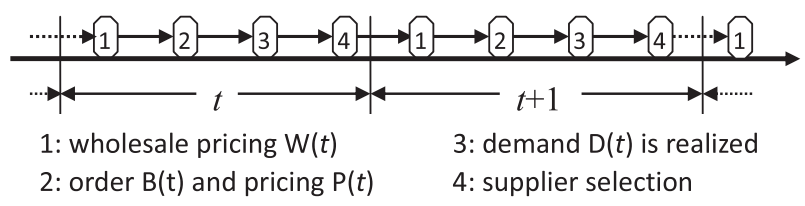

Fig. 2. Decision sequences over time steps, which can be divided into four stages in each time step.

between the individual return and the mean return, where $\operatorname{std}\left(\gamma_{i}\right)$ is the standard deviation of $\gamma_{i}$. Now, we introduce a valuation function of current "buyer-supplier" (i.e., retailer-supplier in this context) relationship,

$$
z_{i j}= \begin{cases}\frac{\gamma_{i}-\bar{\gamma}}{\operatorname{std}\left(\gamma_{i}\right)}, & \text { if } a_{i j}(t)=1, \\ 0, & \text { if } a_{i j}(t)=0 .\end{cases}
$$

For retailer $R_{i}$, suppose his current supplier is $S_{j_{1}}$. Given another supplier $S_{j_{2}}\left(j_{2} \neq j_{1}\right)$, if

$$
\theta+\rho z_{i j_{1}}-w_{j_{1}} b_{i}(t)<\rho z_{i j_{2}}-w_{j_{2}} b_{i}(t) .
$$

then retailer $R_{i}$ will change his supplier from $S_{j_{1}}$ to $S_{j_{2}}$, where $\rho$ measures to what extent retailers emphasize on "buyer-seller" relationship, and $\theta$ is a reputation cost for changing supplier. Once retailer $R_{i}$ decides to change his supplier, he will choose the one (other than $S_{j_{1}}$ ) such that $\rho z_{i j}-w_{j} b_{i}(t)$ gets its maximal value. According to Eq. (6), he will choose the one with the lowest wholesale price.

\subsection{Decision sequence and simulating process}

According to the definition described above, in this section, we shall model decision sequence in Fig. 2, which can be divided into four stages in each time step: (i) first, suppliers give their wholesale prices; (ii) second, retailers decide how many to order from their own suppliers, and how much to retail for a unit of product; (iii) third, demand is realized; (iv) finally, each retailer will decide whether to change his supplier or not.

However, Fig. 2 does not detail all actions in the network, but sketches our model. In the following, we develop a decisionmaking process for simulation, with which we examine our model.

At the Initial status, we set $t=0$, and fix $\eta, \rho$ both ranging from 0 to 1 , and randomly initialize the following parameters: $c^{p}, c^{h}, W(0), A(0)=\left[a_{i j}\right], i=1, \ldots, n, j=1, \ldots, m$; Second, the wholesale price is determined according to $w_{j}(t+1)=w_{j}(t)+\eta \frac{\Delta \pi_{j}^{c}(t)}{\pi_{j}^{c}} \Delta w_{j}(t), j=1, \ldots, m$; Third, the retailers simultaneously determine their own retailing prices and order quantities by solving the newsvendor problem (Eq. (4)) to get their respective price $b_{i}(t), p_{i}(t), i=1, \ldots, n$; Then, after the stochastic demand for each retailer is realized, such retailers satisfying Condition (7) will decide whether they change their respective suppliers by choosing new ones whose have the lowest wholesale prices for their corresponding retailers. Finally, the suppliers will satisfy the demands ordered by retailers.

\section{Model results and analysis}

In this section, we shall present the model results and analysis about the effect of suppliers' altruism on supply networks. Although we can set an arbitrary number of agents (suppliers and retailers), the number of agents who face a homogeneous market ranges from 20 to 200 [39]. Therefore, in our model, we will examine a supply network with comparatively small number of agents: $n=30, m=10$. The parameters $c_{j}^{p}$ and $c_{i}^{h}$ are distributed uniformly in $[0.5,0.6]$ and $[0.2,0.25]$, respectively, $i=1, \ldots, 30, j=1, \ldots, 10$. For the sake of simplicity, we set $c^{d}=c^{h} / 2$. Initially, each retailer randomly chooses his supplier from all the suppliers with equal probability.

We then set the memory length $\tilde{t}=50$, that is to say, we call a segment if the simulation lasts for 50 time steps. From our simulations, we extract 10,000 segments with different $\eta$ or $\rho^{6}$, both of which increase from 0 to 1 with step 0.01 . Figs. 37 illustrate the evolving results over time with $\eta=0.1, \rho=0.1$. Fig. 3 shows the evolving processes of $w$ of suppliers over time, and Fig. 4 gives corresponding changes of all suppliers' sold quantities. From Fig. 3, we can see that there always exist some wholesale prices which become steady after limited time steps, while others change frequently throughout the simulation. In addition, after those suppliers' pricing strategies get into steady statuses, the suppliers can then be divided into two groups: some with constantly positive sold quantity $Q$, and others with 0 sold quantity for ever which implies death in the network. For retailers, there is only a few who face a zero order quantity for ever because the uncertainty of market and their differential pricing (Fig. 5). Moreover, one can find that, in general, a lower wholesale price $w$ brings to both higher

\footnotetext{
6 Except for $\rho$ and $\eta$, other parameters remain constants in all segments.
} 


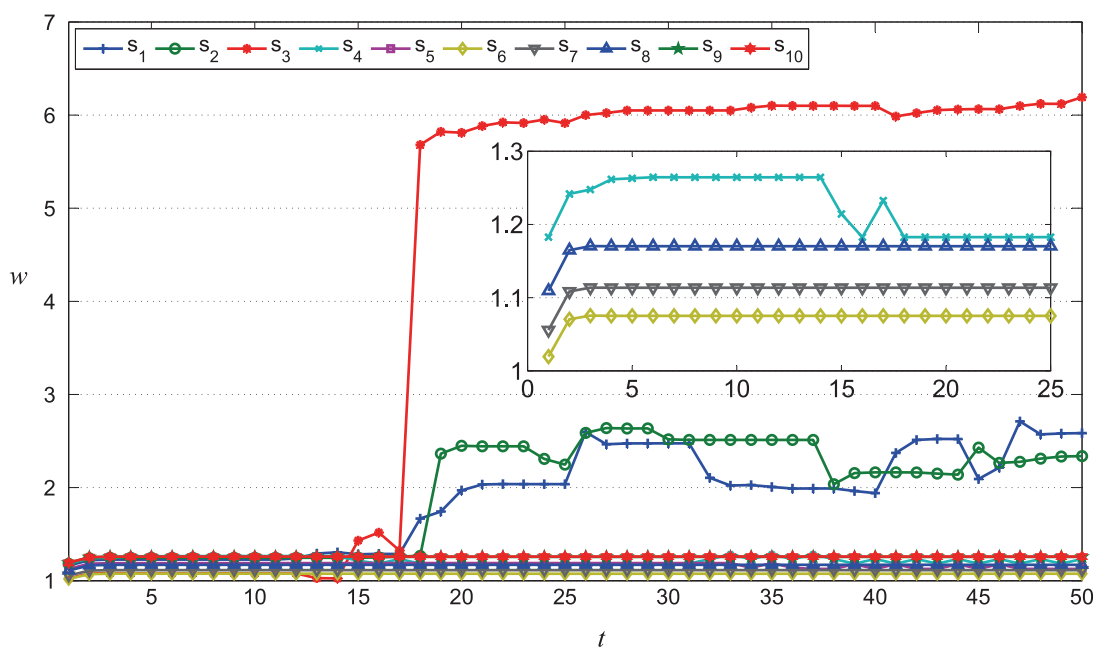

Fig. 3. The wholesale pricing, $w$, evolves over time. The inset shows pricing processes for suppliers $S_{4}, S_{6}, S_{7}$ and $S_{8}$, whose prices change slightly over time (henceforth $s_{j}$ for supplier $S_{j}$ in the figures).

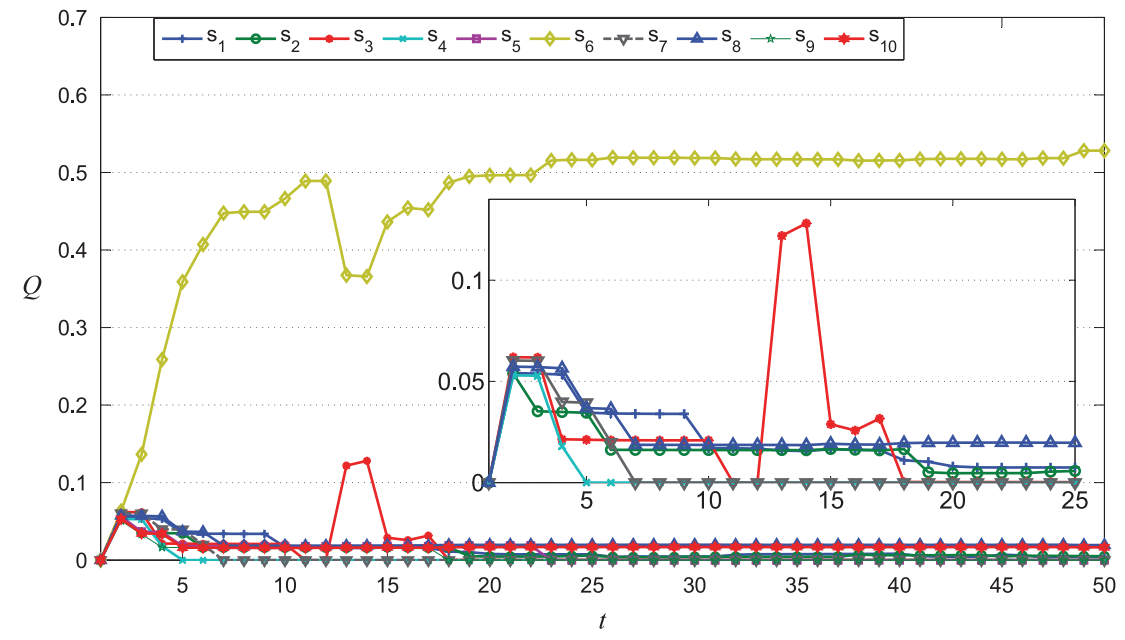

Fig. 4. Sold quantities of suppliers over time, where the inset shows the cases for $s_{1}, s_{2}, s_{3}, s_{4}, s_{7}, s_{8}$. The sold quantity also presents a competitive result among such supply chains supported by different suppliers.

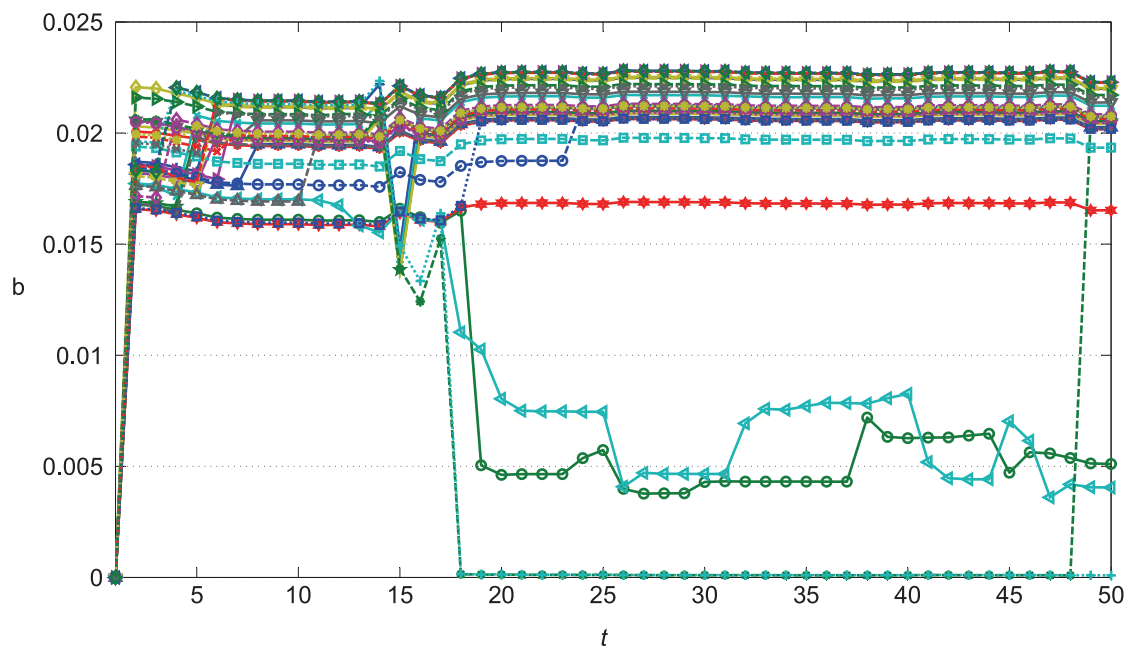

Fig. 5. Order quantities of all retailers over time. The legend of this figure is hidden because of the large number of retailers. However, the overall trend is very clear: most order quantities maintain a slight adjustment over time, while a few change sharply, even decrease to zero for ever. 


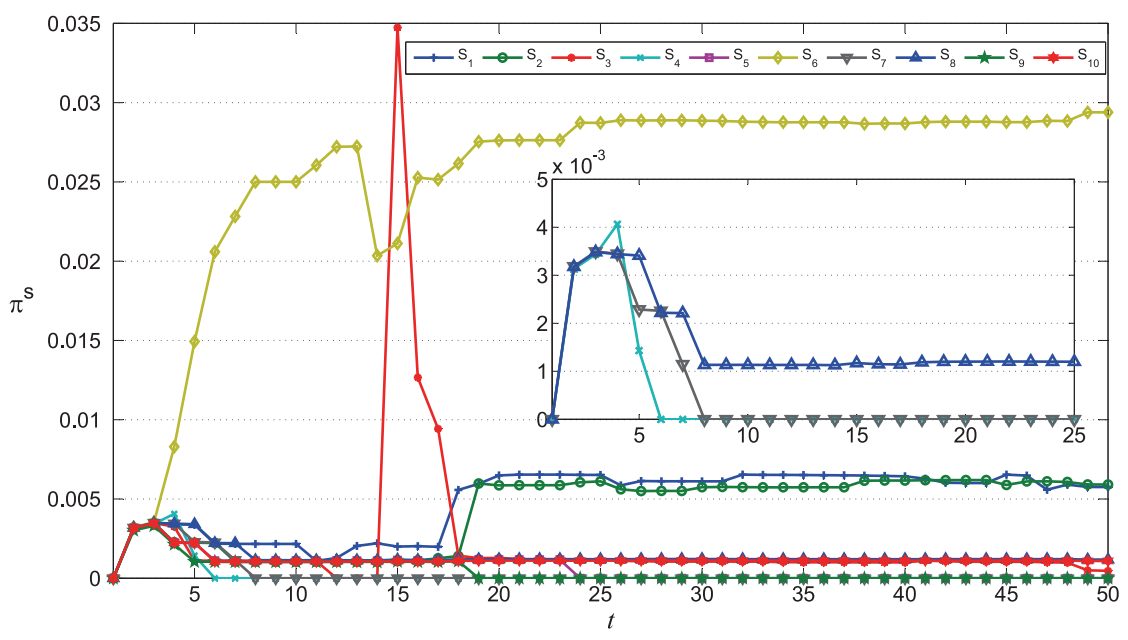

Fig. 6. The evolution of suppliers' profits over time, the inset shows the cases of supplier $j=3,4,7,8$.

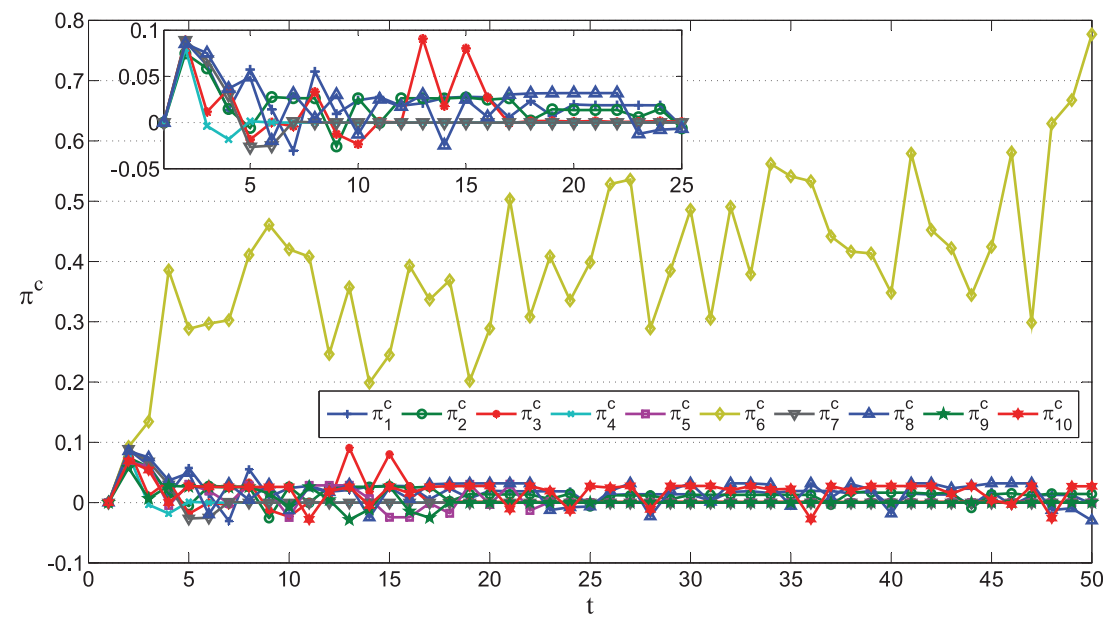

Fig. 7. Supply chains' profits change over time, which are identified by suppliers, that is to say, the chains with the same supplier are calculated together.

sold quantity $Q$ (Fig. 4) and higher profits (Figs. 6 and 7). For instance, suppliers $S_{3}$ faces an significant cut of order quantity from retailers after he lifts his wholesale price at $t=17$, even though an short-term increase before that (from $t=14$ to 17).

Figs. 8 and 9 show the dependency of profits on parameters $\eta$ and $\rho$, respectively. From Fig. 8, we can find an interesting phenomenon: a complete altruism may not be best choice for suppliers. Actually, the suppliers' profits shift concavely with $\eta$. This might suggest that the appropriate regard of others will benefit both himself and his parterres. In our model, $\eta=0.6$ gives the best performance for the suppliers, while $\eta=0.4$ for the whole market. This is caused by the fact that the retailers' profits decrease with $\eta$, which directly results from a sharp decrease of $Q$ (see Fig. 10). However, retailers' profits roughly keep constant when $\eta$ larger than a critical value, e.g., 0.4. Therefore, the supplier's altruistic has little impact on retailers.

In addition, one can find that there is little effect on improving all the agents' profits for large $\rho>0.2$ in Fig. 9, especially for the whole market. However, the retailers' profits increase but suppliers' profits decrease with $\rho$, respectively for small $\rho<=0.2$. This might indicate that the more emphasis the retailers put on the benefit from the "buyer-seller" relationship, the more profits the retailers get, while the less profits the suppliers get.

Furthermore, Figs. 11-13 illustrate the joint effects of both $\eta$ and $\rho$ on the mean profits of supply chains, suppliers, and retailers, respectively. Besides the results above, one can find that suppliers intend to exhibit altruism for $\eta \in[0.4,1]$ (Fig. 12), while a retailer would choose his preference $\rho$ between 0.2 and 1 (Fig. 13). In return, both the network and suppliers would benefit if suppliers limit their preference level $\eta$ in a smaller interval, such as [0.4,0.6] (Figs. 11 and 12). Moreover, the mean profits of both the network and suppliers change slightly with $\rho$ in $[0.2,1]$ (Fig. 11), so does that of retailers with $\eta$ in $[0.4,0.6]$ (Fig. 13). Thus, they might show indifference in partners' preference level in this model. Then, it would benefit for all agents in such an area of preference level, since there is no significant conflicts of incentives. 


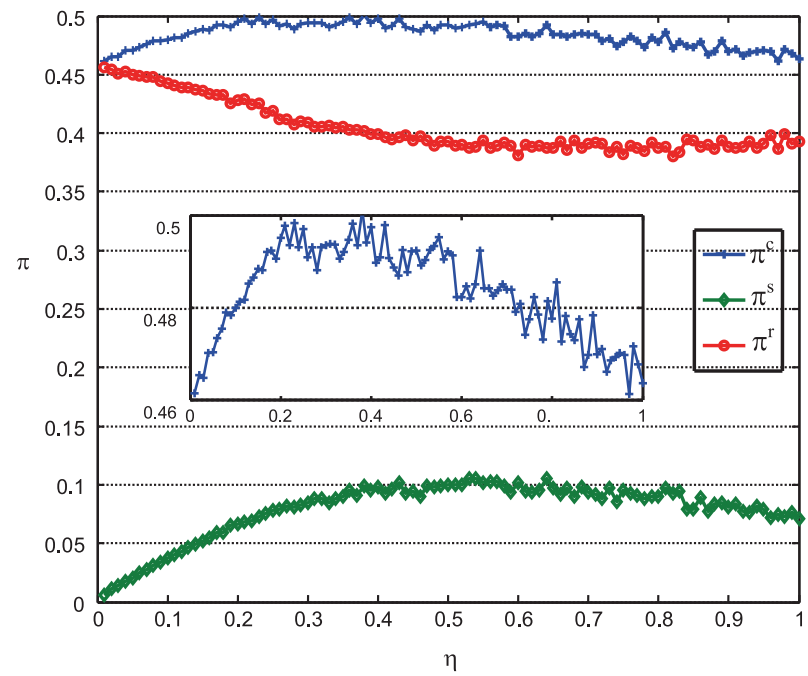

Fig. 8. Profits as a function of suppliers' altruistic preferences, $\eta$. $\pi^{s}$ is an average profit of all suppliers, $\pi^{r}$ is that of all retailers, and $\pi^{c}$ is that of the network, i.e., $\pi^{s}+\pi^{r}$. Each data point is generated by averaging across time steps and $\rho$. The inset shows $\pi^{c}$.

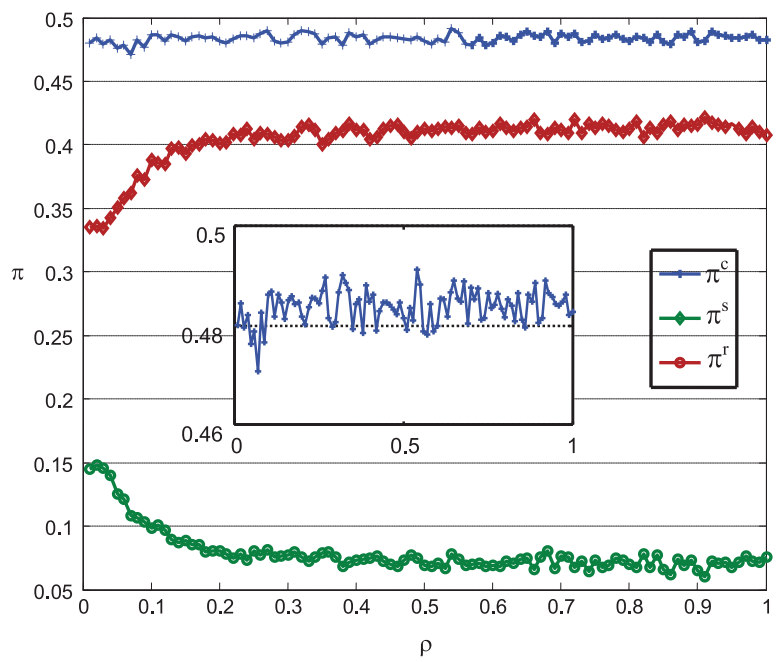

Fig. 9. Profits as a function of retailers' preferences, $\rho . \pi^{s}, \pi^{r}, \pi^{c}$ are the same with that in Fig. 8. Each data point is generated by averaging across time steps and $\eta$. The inset shows $\pi^{c}$.

\section{Conclusions and discussion}

Despite the volume of research on Supply Chain Management, there is only limited attention to such "buyer-seller" relationship in supply networks in the literature. In this paper, we developed an evolutionary decision-making mechanism to characterize suppliers' altruism supported by an agent-based model. The results show that the performances of both suppliers and supply chains are improved with considering altruistic, comparing with noncooperative scenarios. This might suggest that an agent would benefit from his own altruism decision. However, an interesting and reasonable phenomenon is that the profits of both suppliers and chains change nonlinearly with suppliers' altruistic preference in general. Furthermore, they reach their peaks at relatively middle altruistic levels, but not simultaneously. Those findings might indicate that the suppliers have incentives to behave altruistically, but still remain competition with each other, which means a coexistence of cooperation and competition, i.e., coopetition.

There are many altruistic examples in real life. A live case comes from a global executives talk [13]. Alexander Cummings, the president and Chief Operational Officer (COO) of Coca-Cola Company in Africa, recounted one of his successful altruistic decisions. In a market with high inflation and currency devaluation, They had held price for several years to maintain his growth momentum. As a result, their bottlers were struggling to make adequate returns. In order to increase their bottlers' profits, Cummings increased the retail price, which surprisingly improved the profitability for both the company and their bottlers even though they faced a deep drop of volume and market share for six months. 


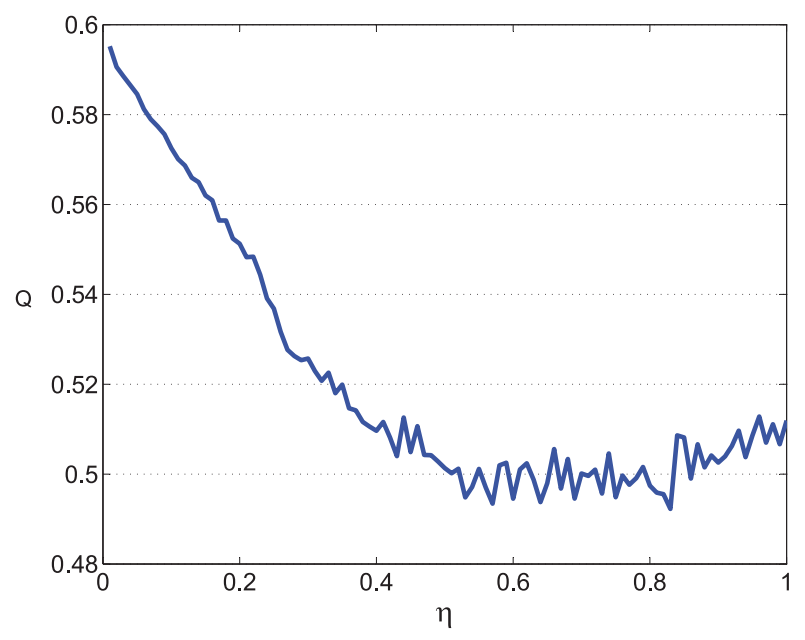

Fig. 10. $Q$ as a function of supplier's altruistic preference, $\eta$.

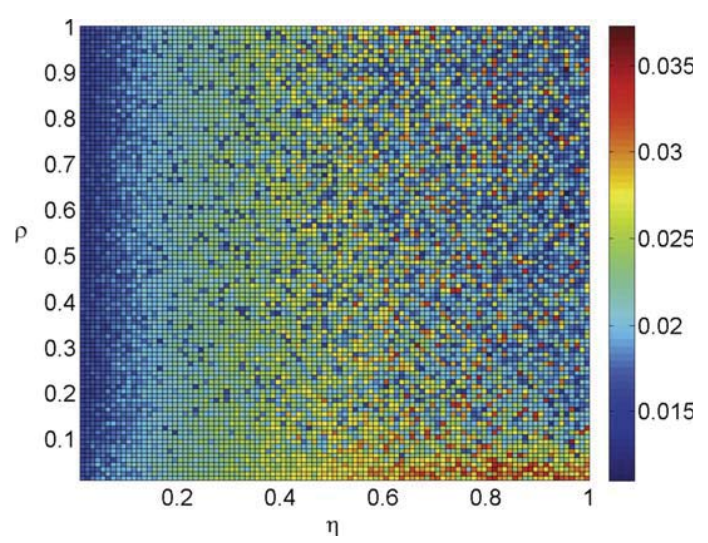

Fig. 11. The mean profits of supply chains shifting jointly with $\eta$ and $\rho$. Each pair of $(\eta, \rho)$ corresponds to a mean profit of all chains over 50 time steps.

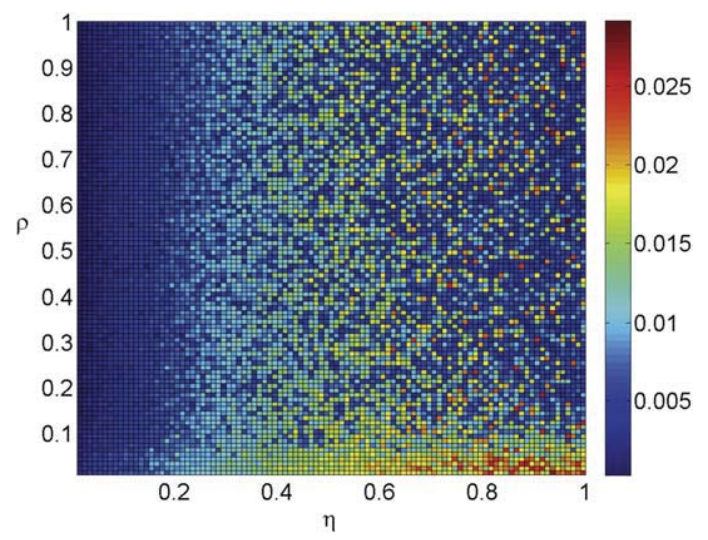

Fig. 12. The mean profits of suppliers shifting jointly with $\eta$ and $\rho$. Each pair of $(\eta, \rho)$ corresponds to a mean profit of all suppliers over 50 time steps.

This paper only provides a simple start point to study the effect of altruism in supply networks, and a couple of open issues remain for the further study. First, in this paper, we focus on the effect of supplier's altruism. Comparatively, the effect of retailers' altruism is lack of study, and it could also be taken into account to investigate how mutual altruism impact the total profit with complex interactions between agents. Second, we only discuss a unique and constant altruism parameter in our model, however, an adaptive altruism mechanism should also be considered to see how altruism works in evolving supply networks. Finally, a supply network structure with multiple echelons, roles and rules is common in reality, so further research should pay more attention to these types of supply networks by developing applicable analysis tools. 


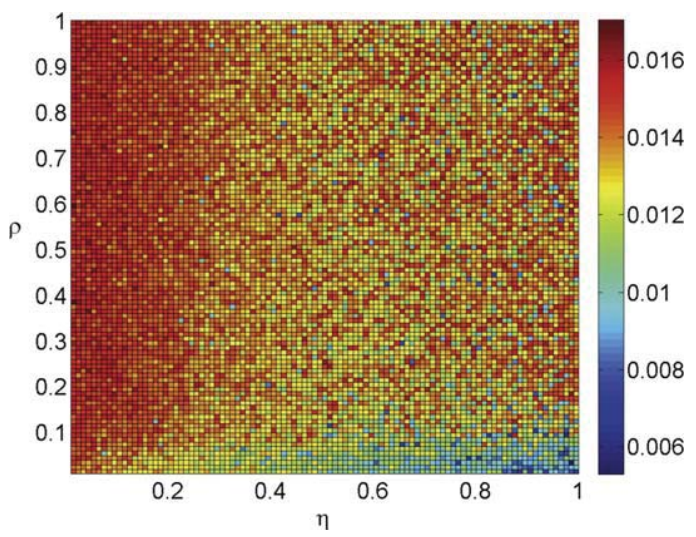

Fig. 13. The mean profits of retailers shifting jointly with $\eta$ and $\rho$. Each pair of $(\eta, \rho)$ corresponds to a mean profit of all retailers over 50 time steps.

\section{Acknowledgments}

This work is partially supported by the Swiss National Science Foundation (Project 200020-121848) and Shanghai leading academic discipline project (S30501). Z. G. acknowledges the National Natural Science Foundation of China (No. 71101092), and the Innovation Program of Shanghai Municipal Education Commission (11YS122). Z.-K. Z., L. L. and T. Z. acknowledge the National Natural Science Foundation of China under Grant No. 90924011.

\section{References}

[1] D. Simchi-Levi, S.D. Wu, Z.M. Shen, Handbook of Quantitative Supply Chain Analysis: Modeling in the E-Business Era, Kluwer Academic Publishers, Boston, 2004.

[2] S.C. Graves, A.G. de Kok, Handbooks in Operations Research and Management Science: Supply Chain Management, North Holland, Amsterdam, 2003.

[3] M. Herty, C. Ringhofer, Physica A 380 (2007) 651.

[4] T. Nagatani, D. Helbing, Physica A 335 (2004) 644

[5] T. Nagatani, Physica A 335 (2004) 661.

[6] M. Laumanns, E. Lefeberobust, Physica A 363 (2006) 24.

[7] S. Pathak, J. Day, A. Nair, W.J. Sawaya III, M. Kristal, Dec. Sci. 38 (2007) 547.

[8] C.M. Harland, Blackwell Encyclopedic Dictionary of Operations Management, Blackwell, 1996.

[9] T.Y. Choi, Y. Hong, J. Oper. Manage. 20 (2002) 469.

[10] A. Nair, R. Narasimhan, T. Choi, Dec. Sci. 40 (2009) 783.

[11] A. Surana, S. Kumarab, M. Greavesc, U.N. Raghavanb, Intl. J. Prod. Res. 43 (2005) 4235

[12] A.-L. Barabási, H. Jeong, Z. Neda, E. Ravasz, A. Schubert, T. Vicsek, Physica A 311 (2002) 590.

[13] O.P. Kallasvuo, G. Jackson, F. Humer, A. Gensler, S. Petrov, A. Klapmeier, A.B. Cummings, D. Aluwihare, Harvard Bus. Rev. 85 (2007) 83. R0701A.

[14] G. Steiner, Business and Society, Random House, New York, 1975.

[15] C.H. Loch, Y. Wu, Manage. Sci. 54 (2008) 1835.

[16] J. Sobel, J. Econ. Lit. 43 (2005) 392.

[17] A. Heifetz, C. Shannon, Y. Spiegel, J. Econ. Theory 133 (2007) 31.

[18] T. Hosoda, S.M. Disney, Automatica 42 (2006) 1301.

[19] E. Fehr, K.M. Schmidt, Quat. J. Econ. 114 (1999) 817.

[20] G.E. Bolton, A. Ockenfels, Amer. Econ. Rev. 90 (2000) 166.

[21] G. Charness, M. Rabin, Quat. J. Econ. 117 (2002) 817.

[22] A. Ugarte, S. Oren, Intl. J. Prod. Econ. 67 (2000) 235.

[23] J. Tooby, L. Cosmides, Proc. Br. Acad. 88 (1996) 119.

[24] P. DeScioli, R. Kurzban, PLoS One 4 (2009) e5802.

[25] H. Schino, Behav. Ecol. 18 (2007) 115.

[26] H. Bester, W. Güth, J. Econ. Beh. Org. 34 (1998) 193.

[27] D.K. Levine, Rev. Econ. Dynam. 1 (1998) 593.

[28] D. Knoch, A. Pascual-Leone, K. Meyer, V. Treyer, E. Fehr, Science 314 (2006) 829.

[29] Y. Bhattacharjee, Science 312 (2006) 1727.

[30] R.L. Trivers, Q. Rev. Bio. 46 (1971) 35

[31] L. Lü, M. Medo, Y.-C. Zhang, Euro Phys. J. B 71 (2009) 565

[32] N.C. Petruzzi, M. Dada, Oper. Res. 47 (1999) 183.

[33] F.Y. Chen, H. Yan, L. Yao, IEEE Trans. Syst. Man. Cybern. A 34 (2004) 450

[34] A. Gürhan Kök, M.L. Fisher, R. Vaidyanathan, Smith, Retail Supply Chain Management, Springer, New York, 2009.

[35] F. Bernstein, A. Federgruen, Manage. Sci. 51 (2005) 18.

[36] G.J. Eidleman, CPA J. 65 (1995) 52.

[37] B. LeBaron, IEEE Trans. Evolut. Comput. 5 (2001) 442.

[38] S. Honkapohj, K. Mitra, J. Econ. Dynam. Control 27 (2003) 1437.

[39] D. Hakimi, B. Montreuil, O. Labarthe, 8th Intl. Conf. Modeling and Simulation (2010). 